\title{
A Switched-Capacitor Interface for Capacitive Pressure Sensors
}

\author{
Mitsuhiro Yamada, Takashi Takebayashi, Shun-Ichi Notoyama, and Kenzo Watanabe, Senior Member, IEEE
}

\begin{abstract}
A switched-capacitor interface for a capacitive pressure sensor is developed which provides a linear digital output. It consists basically of a sample/hold circuit followed by a charge-balancing analog-to-digital converter. The sensor capacitance changes hyperbolically with an applied pressure. To convert the nonlinear capacitance change into the linear digital output, two linearization methods are investigated. In either method, a linear digital output with an accuracy higher than 8-bit is obtained. Because of high accuracy capability and compatible fabrication process, the interface described is best suited for a smart silicon capacitive pressure sensor.
\end{abstract}

\section{INTRODUCTION}

A CCORDING to a 1989 survey, pressure sensors get a $60 \%$ share of the sensor market. This big market, whose annual sales are now over one billion dollars, was already expected in the late sixties for vast applications in pneumatics, medicine, and automobiles. These applications require low cost, mass producible pressure sensors [1]. To meet such a requirement, the integrated sensor consisting of the piezoresistance bridge diffused or ion implanted onto a silicon diaphragm has been developed [2], [3]. This type of pressure sensor features a good linearity, but suffers from the temperature dependence and large power dissipation.

Lower temperature dependence and small power operation can be expected from capacitive pressure sensors [4], [5]. In addition, their sensitivity is 10 to 20 times higher than that of the piezoresistance bridge [6]. However, the measured capacitance change, which is still small compared to the offset capacitance, is comparable with stray capacitances due to packaging and leads. This makes it mandatory to include interface electronics on the sensor chip.

As an interface to meet this requirement, relaxation oscillators were proposed [7]-[9]. These circuits are simple enough to be fabricated by a CMOS process which is compatible with a capacitive pressure sensor. High resolution cannot be expected, however, because they convert the total capacitance of the sensor into frequency. Another promising candidate for the on-chip interface is the switched-capacitor circuit. Of several such interfaces

Manuscript received May 14, 1991; revised September 18, 1991.

M. Yamada and K. Watanabe are with the Research Institute of Electronics, Shizuoka University, Hamamatsu, 432 Japan.

T. Takebayashi and S. Notoyama are with the Product Development Department, SMC Corporation, Soka, 340 Japan.

IEEE Log Number 9105796.
[10]-[12], those based on the charge-balancing or oversampling principles will be best suited for the capacitive sensor, because offset capacitance cancellation and digital encoding of capacitance change can be realized with a minimum device count [13], [14].

The response of the capacitive pressure sensor is highly nonlinear. This poses another problem to the on-chip interface. This paper endeavors to provide the linearization function in the interface circuit. To this aim, nonlinear behavior of a capacitive pressure sensor is first examined. From this examination, it is concluded that linearization is possible only with digital techniques. As a result, the interface must include the digital encoding function. A switched-capacitor charge-balancing analog-to-digital (A/D) converter is adopted for this application based on work done by [13]. Two approaches to linearization appropriate for the charge-balancing A/D converter are then investigated. In either approach, satisfactory results from prototype interfaces are obtained.

\section{Electrical Model of Capacitive Pressure SENSORS}

A general structure of a capacitive pressure sensor is shown in Fig. 1. When the diaphragm, which is anodically bonded to a glass or silicon substrate, bends due to a pressure, the capacitance of a chamber changes.

Assuming a circular diaphragm with the geometric dimensions shown in Fig. 1, the elastic deflection of the diaphragm restrained around its circumference under the pressure $P$ is given by

$$
d(r)=\frac{3}{16} P \cdot \frac{1-\mu^{2}}{E h^{3}} R^{4}\left\{1-\left(\frac{r}{R}\right)^{2}\right\}^{2}
$$

where $h, \mu$, and $E$ are the thickness, the Poisson ratio, and the elastic modulus of the diaphragm, respectively. The capacitance of the chamber is then expressed by the following integral:

$$
C(P)=\epsilon_{0} \epsilon_{r} \int_{0}^{R} \frac{2 \pi r d r}{d_{0}-d(r)} .
$$

Performing the integral and some manipulation, we can express the capacitance as a function of the fractional pressure $x$ :

$$
C(x)=C_{0} \frac{1}{\sqrt{x}} \tan h^{-1} \sqrt{x},
$$




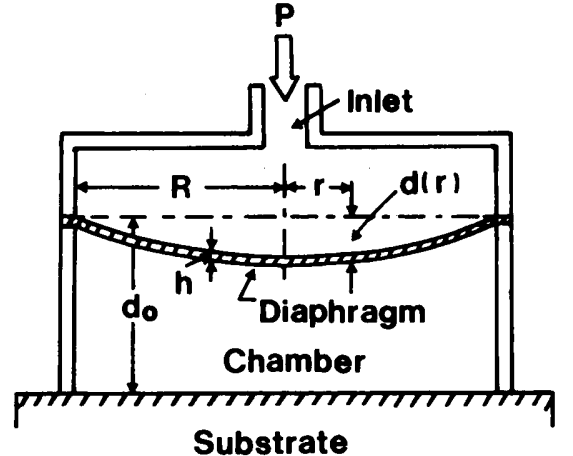

Fig. 1. A structure of a capacitive pressure sensor.

where

$$
\begin{aligned}
x & =\frac{P}{P_{m}}(\leq 1), \\
P_{m} & =\frac{16}{3} \cdot \frac{E}{1-\mu^{2}} \cdot \frac{h^{3} d_{0}}{R^{4}},
\end{aligned}
$$

and

$$
C_{0}=\frac{\pi R^{2} \epsilon_{0} \epsilon_{r}}{d_{0}}
$$

It is clear physically that $P_{m}$ is the maximum allowed pressure which causes a center deflection equal to the chamber depth $d_{0}$, and $C_{0}$ is the offset capacitance when $P=0$. Expanding (3) into a Taylor series, we have

$$
\begin{aligned}
C(x) & =C_{0} \sum_{n=0}^{\infty} \frac{x^{n}}{2 n+1} \\
& \cong C_{0} \frac{1-(2 / 3) x}{1-x}+O\left(x^{2}\right),
\end{aligned}
$$

where $O\left(x^{2}\right)$ denotes the residual term of second-order small.

Some of the practical sensors use stepped diaphragms to increase a pressure sensitivity, and some use square diaphragms fabricated by anisotropic etching techniques. Despite these different structures, it is found experimentally that their capacitance versus pressure characteristics are hyperbolic and can be described by

$$
C(x)=C_{0} \frac{1-\alpha x}{1-x}=C_{0}+\Delta C(x)
$$

where

$$
\Delta C(x)=C_{0} \frac{1-\alpha}{1-x} x
$$

Fig. 2 compares the capacitances measured and approximated by $(8)$ for three capacitive pressure sensors. The parameters $\alpha$ and $P_{m}$ of each sensor are determined by the measured capacitances. Error between the measured and approximated values is less than $1 \%$. Therefore, a capacitive pressure sensor can be regarded reasonably as a nonlinear capacitor described by (8).

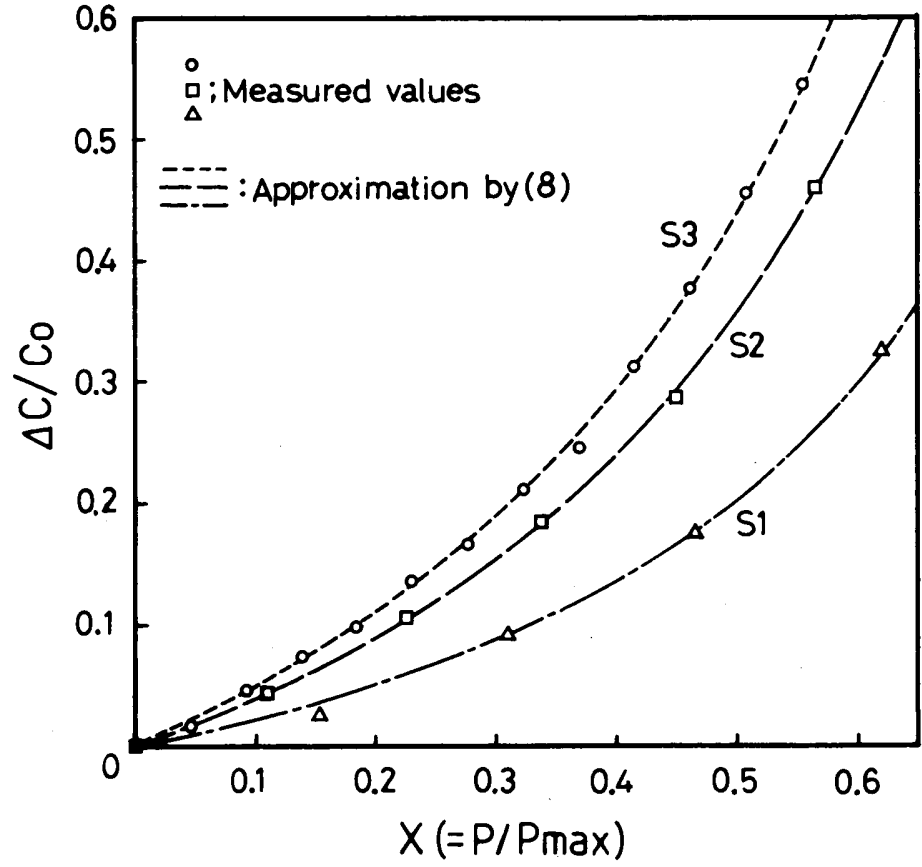

Fig. 2. Capacitance change of diaphragm sensors as a function of applied pressure.

\section{INTERFACE}

A basic configuration of the interface circuitry is shown in Fig. 3. Here, $C(x)$ denotes the sensor capacitance. The circuit operation is divided into the sample and hold states. In the sample state, the sample/hold $(\mathrm{S} / \mathrm{H})$ stage senses the sensor capacitance to its proportional charge $Q(x)$. During the subsequent hold state, the differential integrator (DI) and the comparator (CP) quantize the charge $Q(x)$ held by the S/H stage with respect to the reference charge $Q_{r}$. The principle of quantization is based on the charge balance. The counter (CNT) stores the quantized result. Each block will be next described in more detail.

Fig. 4 shows the circuit diagram of the $\mathrm{S} / \mathrm{H}$ stage. Here, $\phi$ and $\bar{\phi}$ are the nonoverlapping two phase clocks, $\phi_{S}$ and $\phi_{H}$ are the state signals discriminating between the sample and hold states, and $V_{r}$ is a reference voltage. In the $\phi=$ "1" phase of the sample ( $\phi_{S}=$ " 1 ") state, the sensor capacitance is charged to the voltage $V_{r}$. In the next $\bar{\phi}=$ " 1 " phase, the charge amplifier formed by op-amp $A_{1}$ transfers the charge $Q(x)\left(=C(x) V_{r}\right)$ stored in $C(x)$ into the capacitor $C_{s}$, producing the output voltage

$$
V_{0}(x)=-Q(x) / C_{s}
$$

This voltage is stored in the hold capacitor $C_{h}$.

During the subsequent hold state when $\phi_{H}=$ " 1 ", the capacitor $C_{h}$ is connected between the virtual ground node (a) and the output terminal of op-amp $A_{1}$. The hold circuit feeds the charge $C_{s} V_{0}(x)$ to the differential integrator (DI) through the node (b) every $\bar{\phi}=$ " 1 " phase. It is noted that the above operation is insensitive to the offset voltage of op-amp $A_{1}$ and parasitic capacitances between each node and ground [15], because the offset voltage is canceled by that stored in $C_{p}$, and parasitic capacitances are switched between the voltage sources and ground. Fur- 


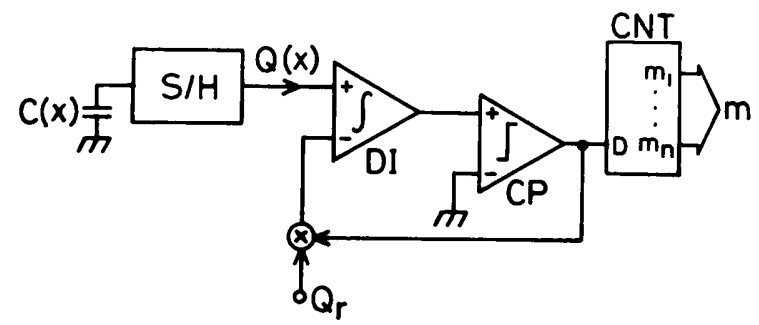

Fig. 3. A block diagram of the interface.
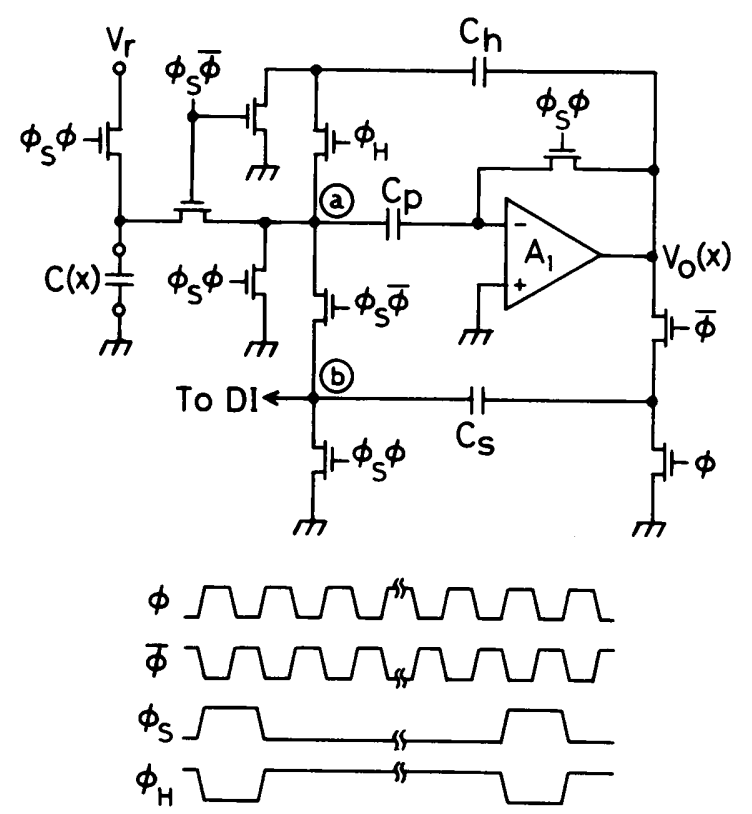

Fig. 4. A circuit diagram of the $\mathrm{S} / \mathrm{H}$ stage.

thermore, the charge fed to the differential integrator is independent of $C_{s}$ and $C_{h}$.

The circuit diagram of the differential integrator and the comparator is shown in Fig. 5(a). The integrator formed by op-amp $A_{2}$ deposits the signal charge $Q(x)$ sent from the $\mathrm{S} / \mathrm{H}$ stage into the feedback capacitor $C_{f}$ every $\bar{\phi}=$ " 1 " phase while extracting the charge $C_{c} V_{c}$ concurrently. Thus, if $V_{c}$ is adjusted such that $C_{c} V_{c}$ be equal to the offset charge $C_{0} V_{r}$ stored in $C(x)$, it operates as the charge circuit to accumulate only the charge produced by an applied pressure into $C_{f}$. When the accumulated charge reaches the reference voltage $Q_{r}\left(=C_{r} V_{r}\right)$, the comparator issues the control signal $\phi_{C}$ for the integrator to extract $Q_{r}$ from $C_{f}$. Repeating this process of charge accumulation and extraction for $2^{n}$ cycles of the two-phase clock, as shown in the timing diagram of Fig. 5(b), the interface counts $\phi_{C}$ using the $n$-bit counter. The count $m$ at the end of the hold state then represents the capacitance change of the sensor quantized by the reference capacitor $C_{r}$ :

$$
\frac{m}{2^{n}}=m_{1} 2^{-1}+m_{2} 2^{-2}+\cdots+m_{n} 2^{-n}=\frac{\Delta C(x)}{C_{r}} .
$$

The quantizing process is insensitive again to the offset voltages of op-amp $A_{2}$ and comparator, and also to parasitic capacitances. The finite gain of op-amp $A_{2}$ has no effect on the quantization, either.

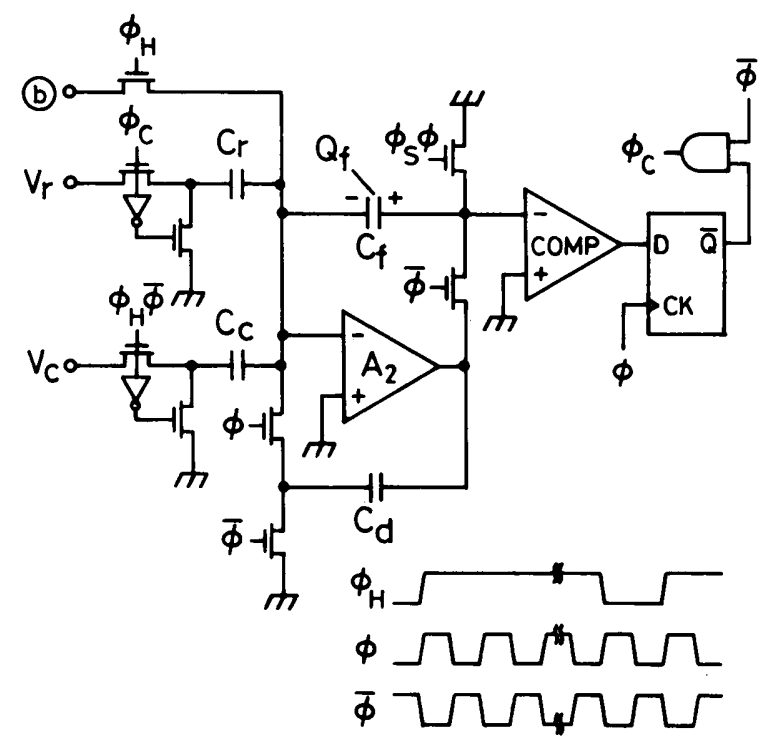

(a)
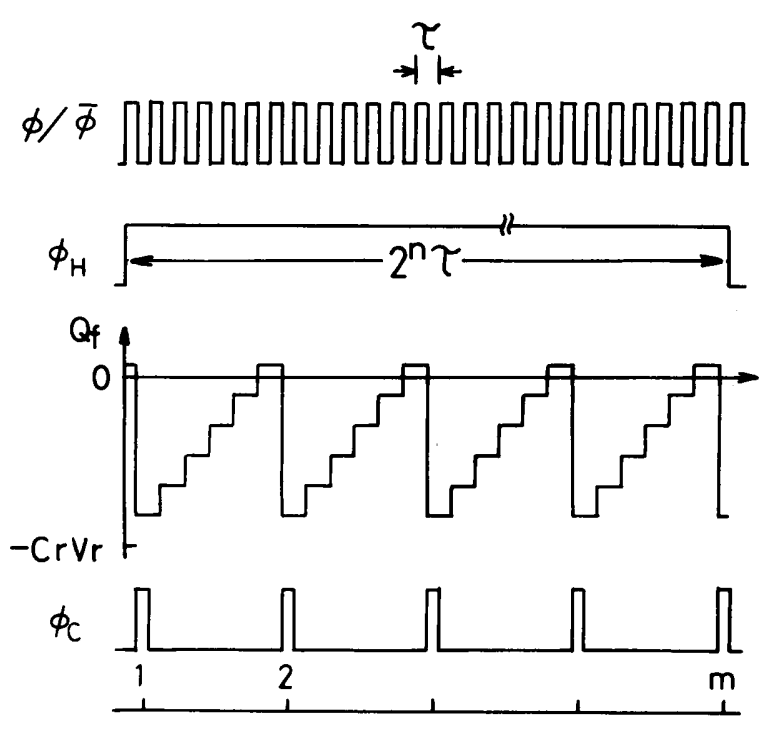

(b)

Fig. 5. A circuit diagram of the differential integrator and the comparator (a) and timing diagram of control signals (b).

\section{LINEARIZATION}

The above-mentioned basic interface converts the capacitance change of the pressure sensor linearly into the digital number. The sensor capacitance changes hyperbolically with a pressure, as described in Section II. Therefore, for the interface to provide the digital output linearly proportional to a pressure, some linearization scheme should be incorporated into the basic configuration.

A well-known circuit technique to linearize a nonlinear sensor is to combine it with a passive linear network. The best approximation to the linear response is then obtained by choosing those circuit parameters of the passive network which make the second derivative of the resultant response as small as possible. This analog technique is not applicable to a capacitive pressure sensor, however, because no inflection point exists in the capacitive versus pressure characteristics. This is evident also by the fact that the capacitance sensitivity, defined as the ratio of the 


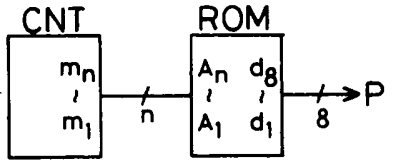

(a)

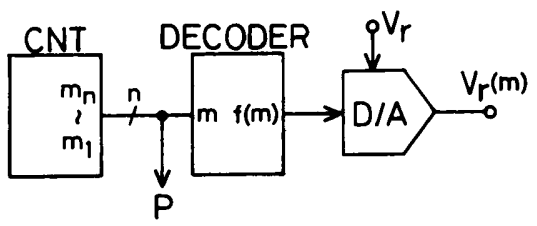

(b)

Fig. 6. Table look-up (a) and nonlinear encoding (b) schemes for linearization.

relative change in capacitance to the relative change in pressure, which measures a nonlinearity, is invariant to a linear transformation. Therefore, linearization is possible only with a digital technique. Two such methods are shown in Fig. 6.

(a) Table look-up method: Storing the conversion table in the ROM and addressing it by the $m-b$ counter in the basic configuration, one can get the digital equivalent of the pressure under measurement. Data $D(m)$ stored in the address $m$ is obtained by (9) and (11):

$$
\begin{aligned}
D(m)= & \frac{m / 2}{1-\alpha+m / 2^{n}} \\
& \quad\left(m=0,1,2, \cdots, 2^{n}-1\right) .
\end{aligned}
$$

A resolution required in most pressure measurements is $1 \%$, and thus $8 \mathrm{~b}$ is enough for the word length. The table address size is determined by the measurement range as follows.

The sensitivity of the capacitance change to the fractional pressure is given by

$$
S_{x}^{\Delta C(x)}=\frac{\partial \ln \Delta C(x)}{\partial \ln x}=\frac{1}{1-x} .
$$

Assume now the fractional pressure in the range from 0 to $3 / 4$ is to be measured. The sensitivity in the lower bound $(x \rightarrow 0)$ is 1 . This implies that the capacitance, and hence $m$, increases linearly with a pressure. The sensitivity in the upper bound, on the other hand, is 4 . Therefore, the table address size should be four times larger than the word length; i.e., $1 \%$ resolution over the range quoted above requires the address size 10 -b wide. This is not a serious problem to the interface because it allows a higher accuracy A/D conversion, but a low sampling rate is inevitable.

(b) Nonlinear coding method: Referring to the charge balance condition, $2^{n} Q(x)=m Q_{r}$; involved in the basic interface configuration, one notices that not the capacitance but the pressure can be linearly encoded by changing the reference charge $Q_{r}$ in a manner similar to $Q(x)$. Since the output $m$ is then the digital equivalent of $x$, this is accomplished by decoding $m$, as shown in Fig. 6(b).
The decoder function $f(m)$ can be derived from (9):

$$
f(m)=\frac{1-\alpha}{(1-m)^{2}} .
$$

This method of linearization requires a D/A converter. Thus, reducing the decoder size is crucial. A technique useful for the reduction is to divide the decoder function $f(m)$ into segments by a piecewise linear approximation. A $1 \%$ resolution can be achieved usually by 32 segments or less. The input $m$ to the decoder is the digital equivalent of the pressure under measurement, and thus $8 \mathrm{~b}$ is enough for most applications. Compared to the table lookup method, this method requires a larger device count, but allows a higher sampling rate.

\section{EXPERIMENTAL Results}

Prototype interfaces were built using discrete components. Op-amp and switches used are LF356 and MC14016, respectively. The clock frequency is $10 \mathrm{kHz}$. Other circuit parameters are: $V_{r}=5 \mathrm{~V}, C_{s}=1 \mathrm{nF}, C_{h}=$ $3.9 \mathrm{nF}, C_{r}=102.2 \mathrm{pF}, C_{f}=1.5 \mathrm{nF}$, and $C_{d}=1.5 \mathrm{nF}$. The capacitive pressure sensor numbered $S 2$ in Fig. 2 is used. Its offset capacitance $C_{0}$ is $50 \mathrm{pF}$. The parameters $\alpha$ and $P_{m}$ calculated using the measured capacitance are 0.64 and $3.56 \mathrm{kgf} / \mathrm{cm}^{2}$, respectively. The pressure applied to the sensor by means of a compressor was measured by a commercially available manometer. The measurement accuracy is $\pm 0.1 \%$ of the $10 \mathrm{kgf} / \mathrm{cm}^{2} \mathrm{FS}$.

Data stored in the look-up table is shown in Fig. 7(a) as a function of the address $m$. The area below the address 65 is saved for the offset capacitance $C_{0}$. The table size is $4 \mathrm{k}\left(=2^{12}\right) \times 8 \mathrm{~b}$. The sampling rate is thus about 2.5 sample-per-second (sps). Fig. 7(b) compares the digital output of the prototype interface displayed on seven-segment LED's with the reading of the commercial manometer. Error between them is less than $1 \%$.

The decoding function $f(m)$ for the nonlinear encoding was divided into 32 segments and stored into a ROM in table form for easy implementation. Data $f(m)$ stored in the table is plotted in Fig. 8(a) as a function of the address $m$. The table size is $256\left(=2^{8}\right) \times 5 b$. The clock frequency is reduced to $7.68 \mathrm{kHz}$, and thus the sampling rate is $30 \mathrm{sps}$. The 5-b D/A converter was built using an R-2R ladder. The pressure displayed by the prototype interface is compared with the reading of the commercial manometer in Fig. 8(b). Both are in good agreement except for the lower and upper bounds, and error between them is within $1 \%$.

\section{Conclusions}

An interface circuit for a capacitive pressure sensor based on the switched-capacitor charge-balancing A/D converter and two linearization techniques were described. Prototype interfaces built using discrete components were also presented to demonstrate the validity of 


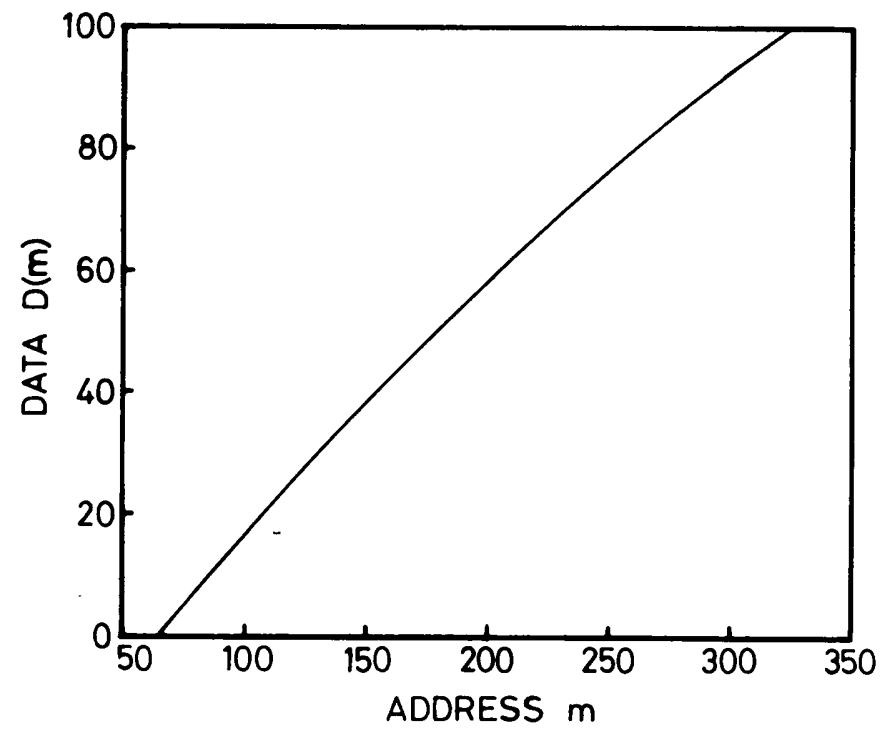

(a)

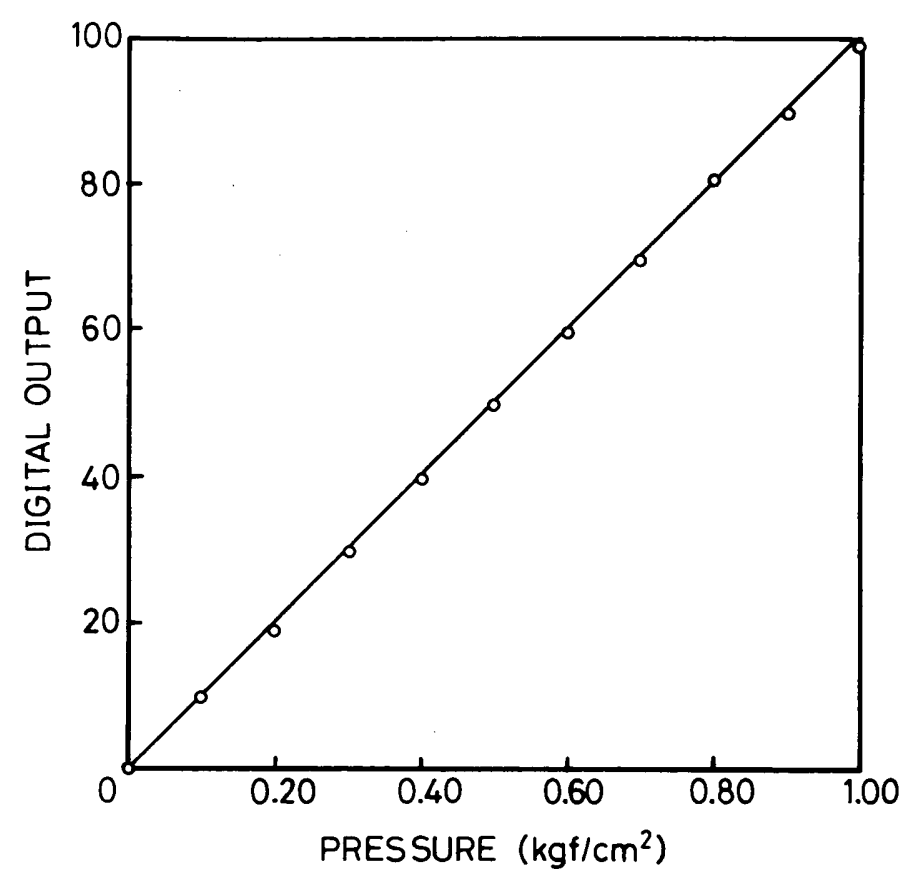

(b)

Fig. 7. Data $D(m)$ stored in the look-up table (a) and the digital output of a prototype interface compared with the reading of a commercial manometer (b).

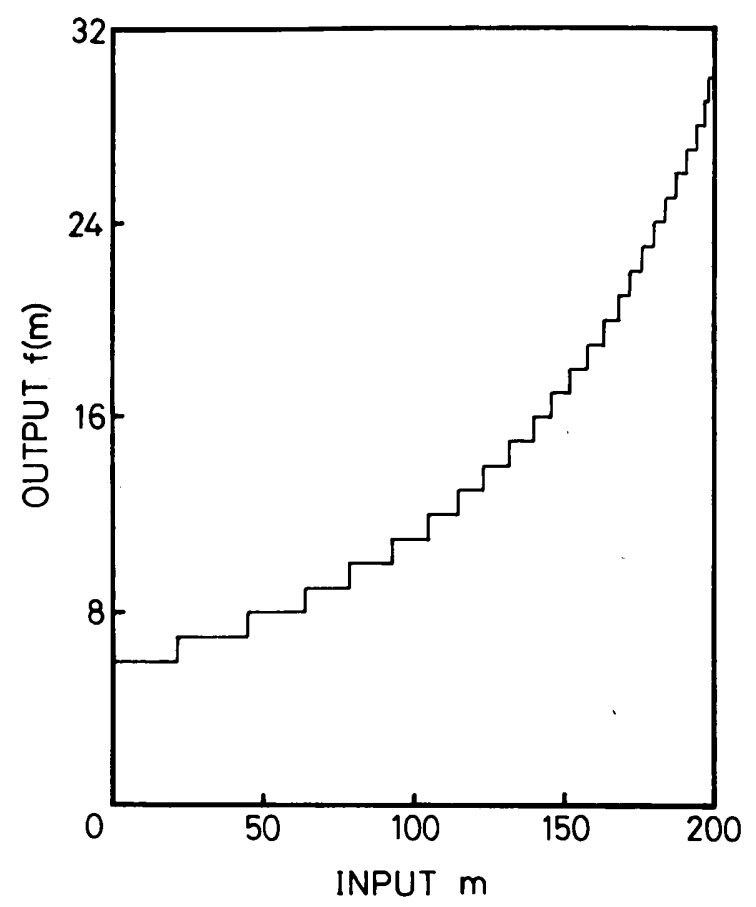

(a)

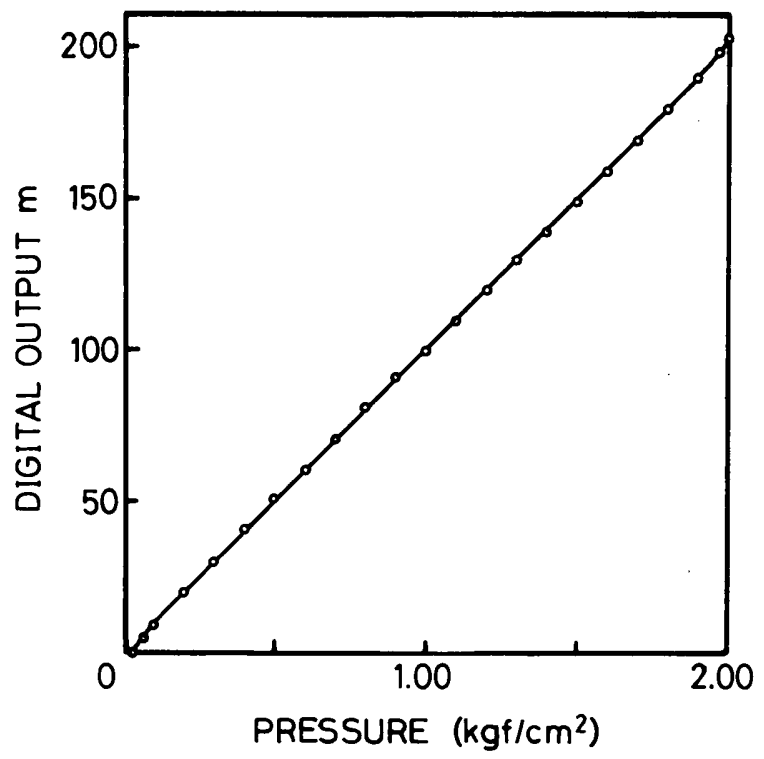

(b)

Fig. 8. The decoder output $f(m)$ stored in a ROM in table form (a) and the digital reading displayed by a prototype interface compared with that of a commercial manometer (b).

the linearization approaches. In realizing the nonlinear encoding approach, the D/A converter using an R-2R ladder was used. Replacing it by a switched-capacitor array will facilitate the monolithic implementation of the interface. This is a future work.

Though different in structure, a great many humidity, displacement, thickness, and flow sensors detect the measurands by their capacitance changes. The interface described herein will find wide applicability in these capac- itive sensors, since it meets such requirements as the onchip implementation, digital output, high sensitivity, and good linearity.

\section{REFERENCES}

[1] S. Middlehoek and S. A. Audet, Silicon Sensors. London: Academic Press, 1989, chap. 3.

[2] Samaun, K. D. Wise, and J. B. Angell, “An IC piezoresistive pres- 
sure sensor for biomedical instrumentation," IEEE Trans. Biomed. Eng., vol. BME-20, pp. 101-109, March 1973.

[3] S. Sugiyama, M. Takigawa, and I. Igarashi, "Integrated piezoresistive pressure sensor with both voltage and frequency outputs," Sensors and Actuators, vol. 4, pp. 113-120, 1983.

[4] Y. S. Lee and K. D. Wise, "A batch-fabricated silicon capacitive pressure sensor with low temperature sensitivity," IEEE Trans. Electron Devices, vol. ED-29, pp. 42-48, Jan. 1982.

[5] W. H. Ko, "Solid-state capacitive pressure transducers," Sensors and Actuators, vol. 10, pp. 303-320, 1986.

[6] S. K. Clark and K. D. Wise, "Pressure sensitivity in anisotropically etched thin-diaphragm pressure sensors," IEEE Trans. Electron Devices, vol. ED-26, pp. 1887-1896, Dec. 1979.

[7] A. Hanneborg, T. E. Hansen, P. A. Ohlckers, E. Carlson, B. Dahl, and $O$. Holweck, "An integrated capacitive pressure sensors with frequency modulated output,' Sensors and Actuators, vol. 9, pp. 345$351,1986$.

[8] J. Neumeister, G. Shuster, and W. von Munch, "A silicon pressure sensor using MOS ring oscillators," Sensors and Actuators, vol. 7, pp. 167-176, 1985

[9] M. Esashi, S. Shoji, T. Wada, and T. Nagata, "Capacitive absolute pressure sensors with hybrid structure," (in Japanese) Trans. IEICE, vol. J73-CII, pp. 461-467, Aug. 1990.

[10] Y. E. Park and K. D. Wise, "An MOS switched-capacitor readout amplifier for capacitive pressure sensors," in Proc. Custom Integrated Circuits Conf., 1983, pp. 380-384.

[11] E. Habekotte and S. Cserveny, "A smart digital-readout circuit for a capacitive microtransducers," IEEE Micro, pp. 44-54, Oct. 1984.

[12] G. J. Yeh, I. Dendo, and W. H. Ko, "Switched-capacitor interface circuit for capacitive transducers," in Dig. Int. Conf. Solid-State Sensors and Actuators, 1985, pp. 60-63.

[13] H. Matsumoto, H. Shimizu, and K. Watanabe, "A switched-capacitor charge-balancing analog-to-digital converter and its application to capacitance measurement," IEEE Trans. Instrum. Meas., vol. IM36, pp. 873-878, Dec. 1987.

[14] A. Cichocki and R. Unbehauen, "Application of switched-capacitor self-oscillating circuits to the conversion of RLC parameters into a frequency or digital signal," Sensors and Actuators A, vol. 24, pp. 129-137, 1990

[15] K. Nagaraj, J. Vlach, T. R. Viswanathan, and K. Singhal, "Switchedcapacitor integrator with reduced sensitivity to amplifier gain," Electron. Lett., vol. 22, pp. 1103-1105, 1986. 